\title{
Hemangiomas cavernosos, su tratamiento con alambres de cobre.
}

\author{
Prof. Dr. Felipe Coiffman, FACS \\ Profesor emérito de cirugía plástica de la Universidad Na- \\ cional de Colombia y de la Universidad de los Andes. Miem- \\ bro de la Academia Colombiana de Medicina. Miembro fun- \\ dador de la Sociedad Colombiana de Cirugía Plástica.
}

\section{Resumen}

La anomalía congénita más frecuente son las lesiones vasculares de la piel. Van desde una simple mácula rosada, hasta tumores deformantes e incapacitantes. La gran mayoría desaparece en el primer año de vida. Otros involucionada espontáneamente en los primeros 6 años. Son más frecuentes en la mujer y en la raza blanca. Hay múltiples tratamientos, pero ninguno da garantías absolutas de curación.

Basado en las observaciones de los campesinos que notaban que un hemangioma cuando era puncionado o se ulceraba, reducía de tamaño, Wang en la China, recomendó puncionar el hemangioma con agujas de cobre y aplicar a ellas ligeras descargas eléctricas. Nosotros modificamos el método. Implantamos simples alambres de cobre que se toman de un cable eléctrico multifilamentoso. Con una aguja recta larga y bajo anestesia local ambulatoriamente, sembramos un emparrillado de alambres en la lesión. A la semana los extraemos. La irritación que produce estimula coagulación intravascular. Al reabsorberse los coágulos, la tumoración desaparece o por lo menos reduce considerablemente de tamaño.

Si es necesario, la piel sobrante se reseca, también bajo anestesia local ambulatoriamente.

El método es muy efectivo, sencillo y económico y puede ser practicado por cualquier cirujano, sea o no cirujano plástico. En 115 casos tratados en los últimos 20 años, no hemos tenido complicaciones.

\section{Palabras claves:}

Hemangiomas. Alambres de cobre. Hemangiomas cavernosos.

\begin{abstract}
Vascular injuries of the skin constitute the most frequent congenital anomaly. They go from a simple pink stain, up to tumors that can cause great deformities. Most of them disappears in the first year of life. Other, suffers spontaneous involution in the first 6 years. They are more frequent in woman and in white race. There are multiple treatments, but none gives absolute guarantees.

On the basis of the observations of rural people, who notice that an injury of this type, when it is punctioned or sore reduces its size, Wang in China, recommended to punction all the venous malformations with needles of copper and to apply on them light electrical unloads. We modify the method implanting simple wires of copper using a straight long needle and under local anesthesia, ambulatory, creating a cross area of wires in the injury. After a week, we extract them. The irritation that we produce stimulates the intravascular coagulation and when hematomas are solved, the malformation disappears or at least it reduces considerably of size. If it is necessary, we resect the remaining skin later under local anesthesia also in ambulatory form.

This method results very effective in our hands, is simple, economic and can be practiced by any surgeon. In 115 cases treated in the last 20 years, we have not had serious complications, except pain and edema in the innmediate postoperatory.
\end{abstract}

\section{Key words:}

Venous malformations, Hemangioma, Cooper wires. 


\section{Introducción}

La anomalía congénita más frecuente en los niños la constituyen las lesiones vasculares cutáneas persistentes (o.6o por cada 1.000 nacimientos vivos).

Los hemangiomas cavernosos son lesiones vasculares formadas por canales venosos dilatados y separados por septos fibrosos. Poseen múltiples vasos alimentadores y de drenaje. Hay en ellos proliferación de células endoteliales y la membrana basal es gruesa. Pueden ser congénitos o desarrollarse a las pocas semanas de vida. Uno de cada diez niños nace con alguna lesión vascular temporal, que generalmente es una pequeña mácula rojiza o un hemangioma plano localizado en la nuca o en cualquier otra región. Generalmente desaparecen al cabo del primer año de vida. No sucede así con los hemangiomas cavernosos, aunque algunos sí involucionan en los primeros 6 años. Son más frecuentes en las mujeres que en los hombres en la proporción de 3:1. Un 60\% se localizan en la cara y en el cuello. Son más frecuentes en la raza blanca que en la raza negra en una proporción de 2:1. (1)

\section{HISTORIA}

Las creencias populares los achacan a fenómenos acontecidos durante la gestación: un susto fuerte de la madre; el ver un animal grotesco; una maldición; un golpe en el vientre gestante; un bebedizo embrujado; la herencia, etc. -Los antiguos anatomistas los llamaban naevus martenus o stigma metroceles. (2)

Ya en la Biblia (Génesis, XXX) se lee que Jacob, hijo de Isaac y de Rebeca, observó la importancia de la herencia en sus rebaños de ovejas. Para mejorar la calidad de éstas, eliminaba a aquellas que tenían defectos en la piel y solo permitía el apareamiento entre los especímenes más sanos y fuertes. Así lograba que sus rebaños fueran más sanos y apetecidos. Además podía controlar el color de la lana.

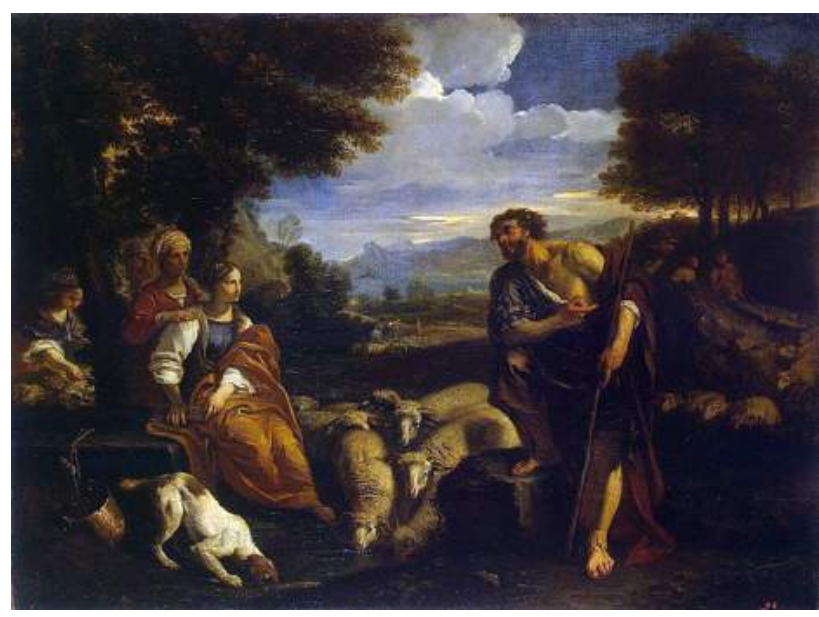

En la antigua Grecia, los espartanos, en su deseo de mantener una raza libre de defectos, a los niños que nacían con malformaciones, entre ellas hemangiomas cavernosos, los arrojaban por los precipicios del Monte Taigeto.

Lesiones del tipo hemangiomatoso fueron descritas por Plinio, El Viejo en su Historia Natural, en los primeros decenios del Siglo I de nuestra era. También Galeno (130-200) y Ambrosio Paré (1.509-1.590) describieron estas lesiones con detalles. Pero fué Virchow (1.821-1.902) quien primero describió la anatomía patológica de los hemangiomas. (3) (4)

Mulliken, Glowacki y Young (1.982; 1.988), en su libro clásico "Vascular Birthmarks" hicieron la clasificación que hoy más se usa. Se basaron en la evolución y en las características endoteliales de estas lesiones. Las dividieron en: 1. Hemangiomas. 2. Malformaciones vasculares y 3. Mixtas.

En el presente estudio nos referiremos solamente a los hemangiomas cavernosos. (5) (6) (7)

\section{TRATAMIENTOS}

Los tratamientos más frecuentes son: ligadura y excisión; ulceración artificial; electrolisis y termocoagulación; terapias esclerosantes inyectadas (morruato sódico, sotradecol só-

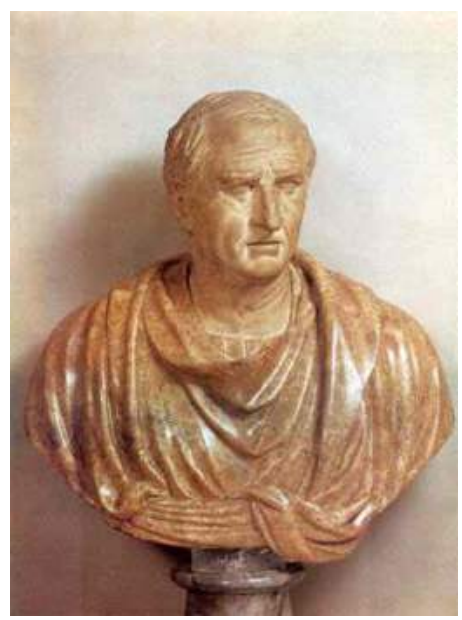
dico, soluciones hiperconcentradas (hipertónicas) de solución salina o de glucosa); irradiación; esperar la involución espontánea; terapia corticoidea; quimioterapia; láser-terapia; embolizaciones; alambres de cobre; maquillaje, etc. Como vemos, los tratamientos son múltiples, pero ninguno de ellos puede garantizar resultados perfectos. (8)

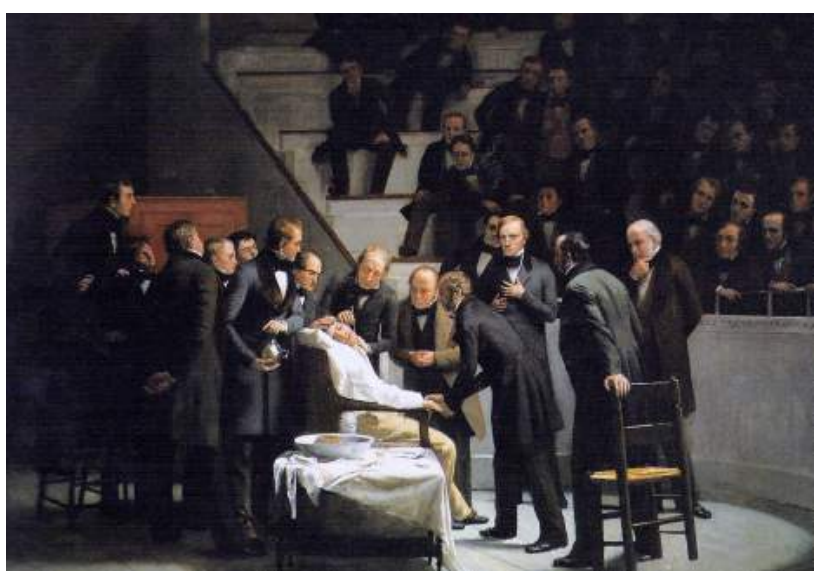


Motivados por el interés en los hemangiomas cavernosos, los hemos estudiado en los dos últimos decenios y hemos encontrado que el tratamiento con alambres de cobre es uno de los más efectivos. La técnica que describiremos la hemos modificado con aportes personales para que sea de fácil aplicación para cualquier cirujano, sea o no cirujano plástico. (Fig. 4) (Fig. 5)

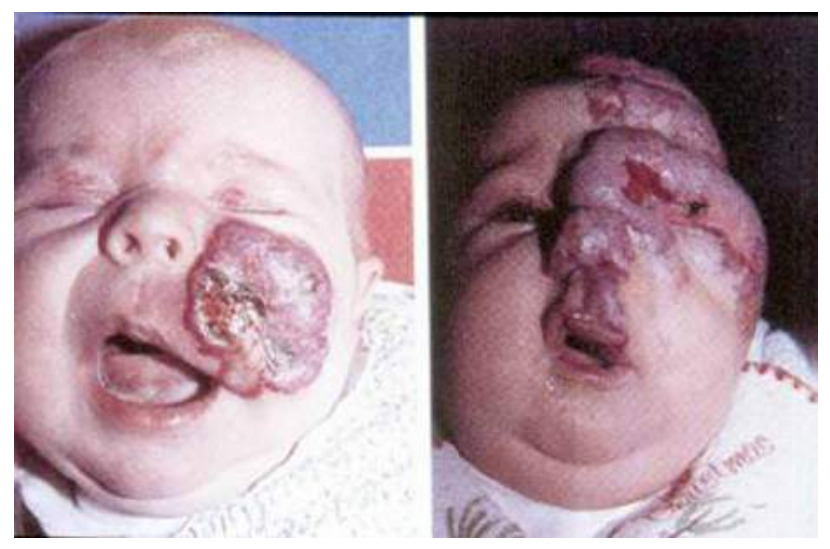

ALAMBRES DE COBRE. TÉCNICA.

De los campesinos aprendimos que cuando un hemangioma cavernoso era accidentalmente puncionado con una espina, por ejemplo, o ulcerado, su tamaño se reducía. (Fig. 6)

Wang (1993) en la China, tuvo la idea de insertar en los hemangiomas agujas de cobre para inducir una coagulación intravascular. Aplicaba bajas descargas eléctricas que al producir una diferencia de potencial entre los polos positivos (vasos sanguíneos) y negativos (agujas), aceleraba la formación de coágulos. En el proceso de reabsorción de estos coágulos, el hemangioma reducía su tamaño. La misma técnica emplearon Calderón (1996), Boo-Chai (1.977) y otros.

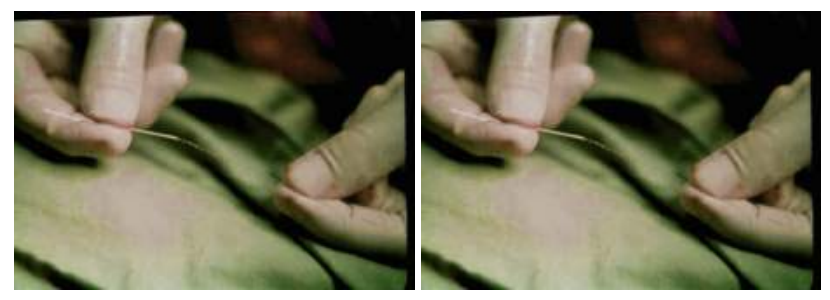

Nosotros modificamos la técnica insertando múltiples alambres delgados de cobre a través del hemangioma. Estos alambres los sacamos de los cables de electricidad multifilamentosos. (Fig. 8) Los insertamos en una aguja larga y los sembramos formando cuadrículas en toda la extensión del hemangioma. Dejamos los dos extremos sobresaliendo para facilitar su extracción, lo cual hacemos a la semana. Usamos anestesia local, ambulatoriamente. (Fig. 9) (10)
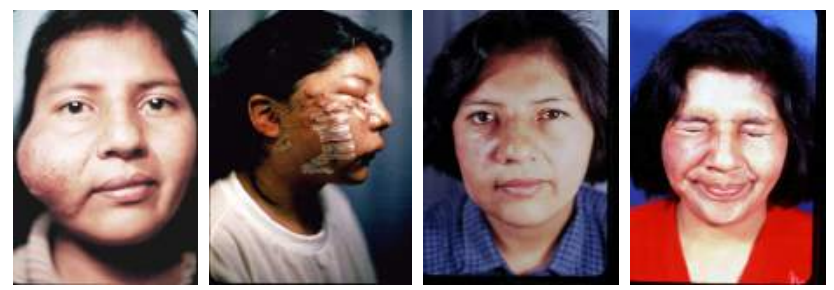

La irritación intravascular que estos alambres producen se manifiesta por el considerable edema de la región. Al cabo de dos semanas comienza a notarse la reducción del hemangioma. Con frecuencia, la piel sobrante debe ser resecada, lo cual se hace también bajo anestesia local ambulatoriamente. (Fig. 10)(11)
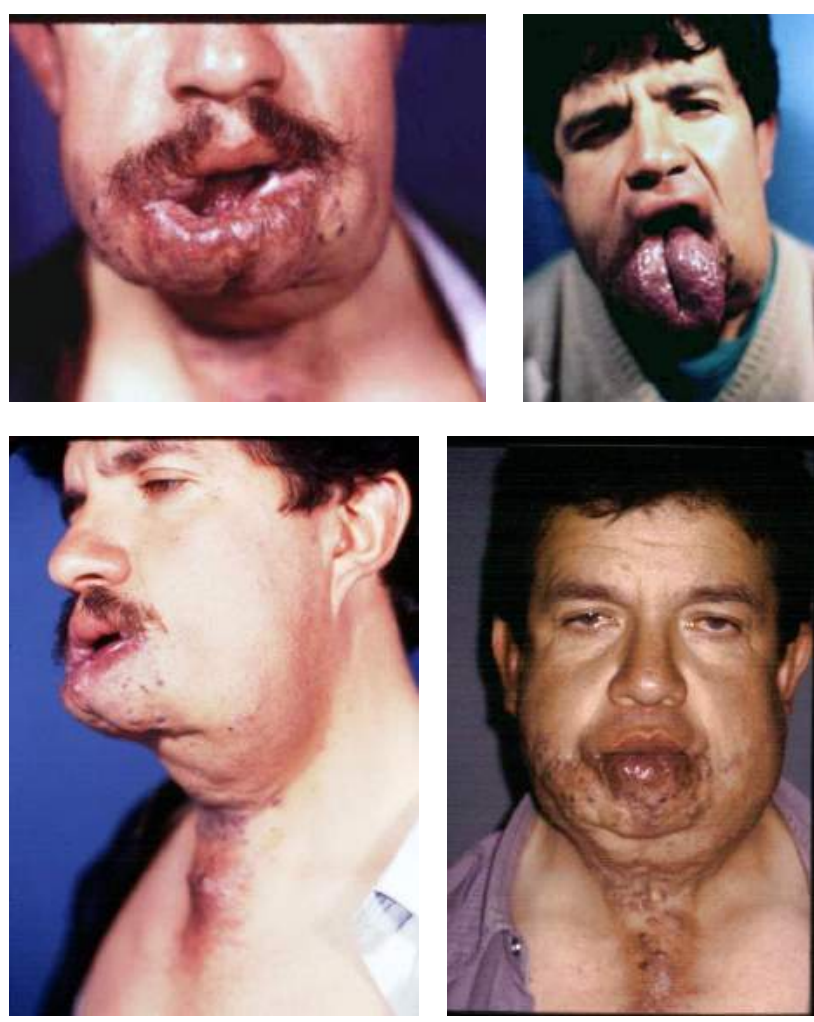

Como vemos, el tratamiento es sencillo, económico y de fácil aplicación. Afortunadamente no hemos tenido complicaciones.

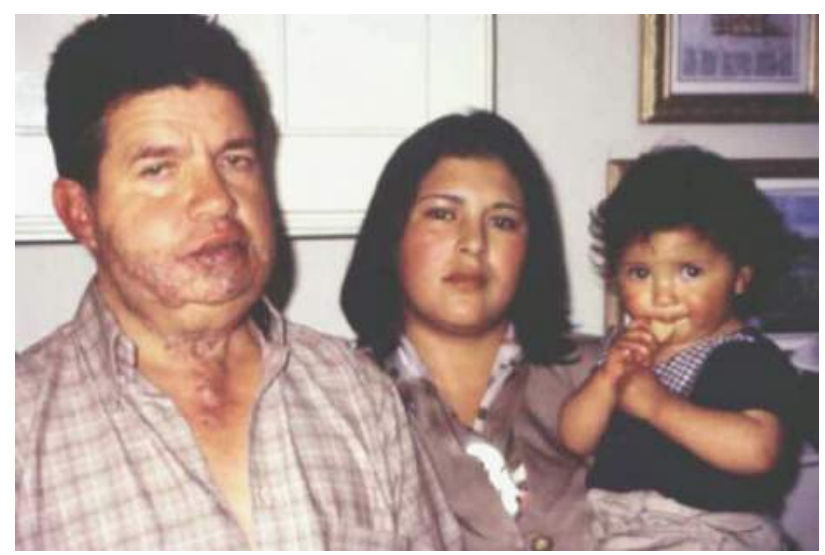




\section{CONCLUSIONES}

La implantación de alambres de cobre en los hemangiomas cavernosos es un método muy eficaz, sencillo, económico y puede ser practicado por cualquier cirujano. Se realiza bajo anestesia local, ambulatoriamente. En nuestros 115 casos, no hemos tenido complicaciones

\section{BIBLIOGRAFÍA}

1. Mulliken, J. B.; Young, A. E.: Vascular Birthmarks. Hemangiomas and Malformation. W. B. Saunders. Co. Philadelphia, 1988.

2. Blondel, J. A.: The Strength of Imagination in Pregnant Women Examin'd: And the Opinión that Marks and Deformities in Children Arise from Thence, Demostrated to Be a Vulgar Error. London: J. Peele, 1727, p. 106.

3. Paré, A.: The Workes of the Famous Chirurgion Ambrose Parey, Book 25. Traslated by Thomas Johnson. London: Thomas Cotes and R. Young, 1634, p. 979.

4. Plinius, Gaius Secundus: Historia Naturales Libro VII, 52.

5. Calderon, D.A.; Toranzo, F. J. M.; Hidalgo, H.J.A. Tratamiento en hemangiomas de la región maxilofacial por medio de electrombosis con agujas de cobre y cirugía. Rev. A.D.M. 1996, 53 (1) 50-53.

6. Wang D.: Retained copper needles for the treatment of cavernous hemangioma. Rev. Zhonghua Zheng Xing Shao Shan Wai Ke Za Zhi 1993; 9: 321-396

7. Boo-Chai, Khoo. Therapeutic coagulation induced in cavernous hemangioma by use of percutaneous copper needles. Plast. Reconst. Surg. Sept. 1977.

8. Pérez, O.; Pérez, C.: Angiodisplasias congénitas. En "Coiffman. Cirugía Plástica, Reconstructiva y Estética”. Tercera Edición. 1er Tomo. Pág. 358. Amolca Editores, 2006.

9. Coiffman, F. (Editor) en "Coiffman. Cirugía Plástica, Reconstructiva y estética”. Tumores precancerosos y seudocancerosos de la piel. $2^{\underline{a}}$ Ed. Pág. 249. Masson-salvat. Barcelona, 1994.

10. Pérez, O.: Hemangiomas. Linfangiomas y Síndromes asociados. En “Coiffman, F. (Editor) Cirugía Plástica, reconstructiva y estética. 2ª Ed. Pág. 259. Masson-Salvat, Barcelona, 1994 .

11. Coiffman, F. Tratamiento de las malformaciones venosas con alambres de cobre. Rev. Cir. Pl. Iberolatinoamericana. Vol. 7. No. 2, pág. 155-160. Mayo-Junio, 2011.
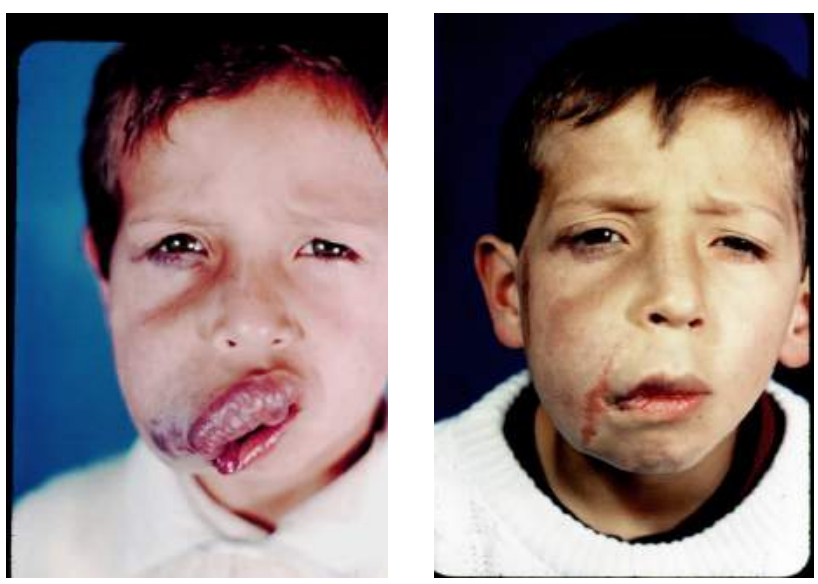

Niño con un gran hemangioma cavernoso en mejilla y labio superior. Se combinó el tratamiento con resecciones parciales. B. El mismo paciente después de 3 años.
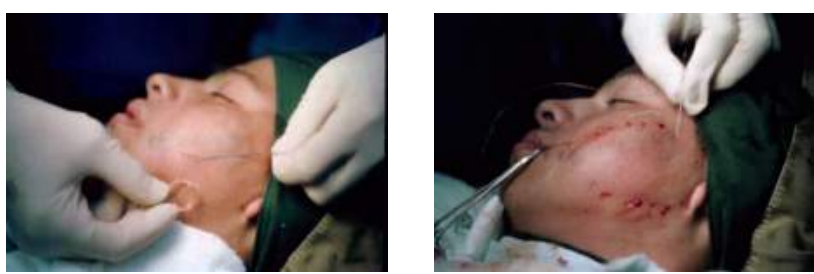

En la imagen izquierda vemos que la aguja atraviesa el hemangioma de la mejilla de un extremo al otro. En la imagen derecha Los alambres se entrecruzan formando un emparrillado. Se dejan los extremos expuestos para su extracción.
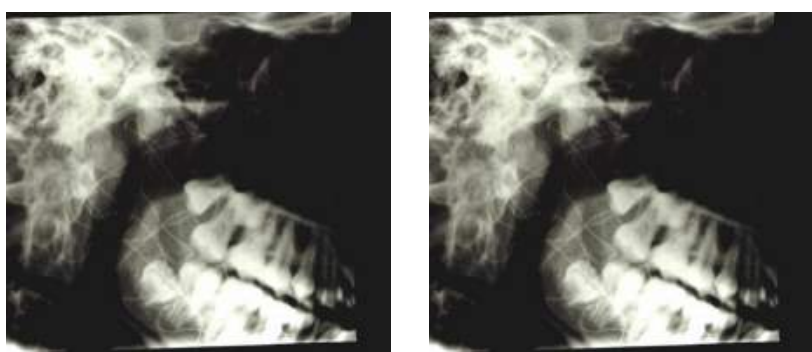

Radiografía mostrando el emparrillado de alambres. E. El edema, como respuesta a la inflamación, es intenso y se produce desde el segundo día.
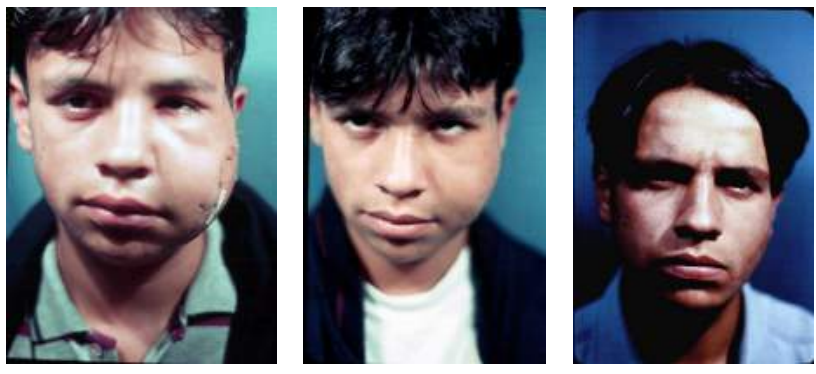

Cambio notorio en el aspecto del paciente. Obsérvese el hemangioma en la mejilla izquierda. El mismo paciente un año después. Nótese la simetría de la cara. 